\title{
Cardiovascular and vasoactive hormone responses to bladder distension in spinal and normal man
}

\author{
H Krum FRACP, ${ }^{1}$ W J Louis MD FRACP, ${ }^{1}$ D J Brown MD FRACP,${ }^{2} \mathrm{~S}$ J Clarke \\ FRACS ${ }^{3}$ J A Fleming FFRACS,${ }^{4}$ L G Howes PhD FRACP ${ }^{1 *}$ \\ ${ }^{1}$ Department of Clinical Pharmacology and Therapeutics, ${ }^{2}$ Spinal Injuries Unit 2 , \\ ${ }^{3}$ Department of Urology, ${ }^{4}$ Department of Anaesthetics, Austin Hospital, Heidelberg, \\ Victoria, 3084, Australia.
}

\begin{abstract}
Many patients with high spinal cord injury experience exaggerated blood pressure rises in response to bladder distension. To examine the humoral mechanisms associated with these responses, ECG heart rate, blood pressure and vasoactive hormone levels were measured at baseline and during bladder distension following slow bladder filling in 23 subjects: 9 high spinal lesion patients, 7 low spinal lesion patients and 7 normal control subjects. Systolic blood pressure rose significantly during bladder distension in the high spinal lesion group by an average of $56 \mathrm{~mm} \mathrm{Hg}(48 \%)$ and diastolic blood pressure rose by $22 \mathrm{~mm} \mathrm{Hg}(47 \%)$, while heart rate fell by a mean of 7.4 beats per minute $(15 \%)$. By contrast, neither systolic or diastolic blood pressure nor heart rate changed significantly during bladder distension in the low spinal lesion or normal control group. There were no significant changes in plasma levels of noradrenaline, renin, aldosterone, vasopressin, arginine, or atrial natriuretic peptide during bladder distension to account for the blood pressure rise in the high spinal lesion group. These findings suggest that humoral mechanisms are unlikely to play a major role in the mediation of pressor responses to bladder distension in high spinal lesion patients.
\end{abstract}

Key words: quadriplegia; bladder distension; catecholamines; autonomic hyperreflexia; blood pressure.

\section{Introduction}

Many quadriplegic spinal cord injury patients develop marked blood pressure rises in response to bladder distension, possibly as a result of increased sympathetic activity. Bladder distension has been observed to provoke the syndrome of autonomic hyperreflexia which is a feature of high spinal lesion patients. In order to determine the relative contribution of alterations in vasoactive hormone levels to these exaggerated blood pressure rises, simultaneous measurement of bladder pressure, blood pressure and vasoactive hormone levels before and during bladder stimulation was performed in quadriplegic spinal cord injury patients with levels above T5 and compared with

\footnotetext{
* Correspondence.
}

paraplegic patients with levels below T5. In addition, because little is known regarding haemodynamic and humoral responses to bladder stimulation in normal subjects, a group of patients without spinal cord injury were also studied.

\section{Methods}

\section{Subjects}

Twenty-three subjects were studied during routine urodynamic testing: 9 patients with high levels of spinal cord injury (above T5), 7 with low levels of spinal cord transection (below T5) and 7 patients without spinal cord injury. Spinal cord injury patients with recent episodes of autonomic hyperreflexia were excluded from the study. The spinal cord injury patients were all at least 3 months post injury, and were not taking 
drugs known to affect the sympathetic nervous system. Two patients in each of the low and high spinal cord injury groups were taking anticholinergic medication, but this therapy was stopped 48 hours prior to the study. No smoking or caffeine consumption was permitted on the day of the study.

Non spinal subjects in the study were volunteers who were referred for urodynamic evaluation of conditions not primarily affecting bladder function: 4 with urge incontinence (detrusor instability), one post vaginal repair, one with enuresis, and one with poor stream and dribbling. Non spinal subjects undergoing urodynamic testing for possible neurologically related bladder dysfunction were excluded from the study, as were patients with concurrent urinary tract infection.

Fully informed consent was obtained from all subjects and the protocol was approved by the Austin Hospital Ethical Review Committee.

\section{Urodynamic study techniques}

Patients were studied during routine urodynamic testing. All patients were placed in a recumbent position and were simultaneously catheterised urethrally with two fine Portex cannulae, external diameter $1.4 \mathrm{~mm}$. The bladder was distended by infusing sterile fluid through one cannula at a constant rate via a Harvard infusion pump. ${ }^{1}$ The rate varied from $4 \mathrm{ml} /$ minute to $20 \mathrm{ml} /$ minute with lower rates being used in patients with greater somatic reflex activity. Measurements were taken with the patients in the supine position. The intravesical pressure was measured via the second cannula which was connected to a Sanborn pressure transducer and recorded on a Hewlett Packard 4-channel electronic recorder. The transducer and recorder were calibrated in $\mathrm{cm}$ of water pressure and the reference point was the upper border of the synthesis pubis, as recommeded by the Standardization Committee of the International Continence Society. Rectal pressure was simultaneously recorded using a rectal probe, which provided a value for total abdominal pressure. The intravesical pressure was calculated as measured bladder pressure minus rectal pressure.
Measurements of blood pressure and vasoactive hormone levels were performed prior to commencement of the study and at that time of 'maximal' bladder distension, defined as the first of the following to occur: spontaneous detrusor contraction, maximum resting intravesical pressure, intravesical volume reaching $750 \mathrm{ml}$ or symptoms of autonomic hyperreflexia (systolic blood pressure rise of greater than $30 \mathrm{~mm} \mathrm{Hg}$ or diastolic blood pressure rise of greater than $20 \mathrm{ml} \mathrm{Hg}$ associated with sweating, headache or flushing).

These criteria were selected to avoid the confounding factor of pain from bladder overdistension which may influence pressor and neurohumoral responses to bladder filling.

Blood pressure and heart rate measurement Blood pressure monitoring was performed using Finapres, a non invasive method of blood pressure monitoring enabling beat to beat measurement of blood pressure based on a principle first described by Penaz ${ }^{2}$ and applied by Wesseling and co-workers. ${ }^{3}$ It has been well validated in a large number of studies against intra-arterial blood pressure monitoring. ${ }^{4,5}$ The instrument operates through a small finger cuff equipped with an infrared photoplethysmograph to measure the arterial blood volume under the inflatable cuff. The accuracy of the Finapres was validated by simultaneous correlation at baseline to manual sphygmomanometer readings in the same arm. The Finapres non invasive continuous blood pressure monitor measured blood pressure throughout the period of bladder filling.

Heart rate was monitored by a 3-lead electrocardiographic recording. Blood pressure, heart rate and intravesical pressure were graphed simultaneously on a multichannel recorder.

\section{Measurement of Vasoactive Hormone Levels}

A venflon cannula was inserted into the patient's antecubital vein prior to commencement of the study. The patient then lay supine for 15 minutes after which time $30 \mathrm{ml}$ of blood was taken for measurement 
of baseline hormone levels. A further $30 \mathrm{ml}$ sample was taken during the time of 'maximal' bladder distension for measurement of plasma noradrenaline, plasma renin concentration, plasma aldosterone, plasma vasopressin and plasma atrial natriuretic factor.

Blood was collected into chilled lithium heparin tubes and placed immediately on ice. Plasma was separated immediately by centrifugation for 10 minutes at $1,000 \mathrm{~g}$ at $4{ }^{\circ} \mathrm{C}$. The samples were stored at $-80^{\circ} \mathrm{C}$ and assayed within 4 days. Noradrenaline was extracted from the plasma using a combined cation-exchange-alumina technique using dihydroxybenzylamine as an internal standard. ${ }^{6,7}$ This technique was subsequently modified by washing the alumina with sodium carbonate before acid elution of catecholamines. ${ }^{8}$

Plasma renin concentration was measured by an enzyme kinetic method (zero order) in which Angiotensin I generated at $37^{\circ} \mathrm{C}$ was measured by radioimmunoassay and corrected to $\mathrm{ng} / \mathrm{ml} / \mathrm{hr} .{ }^{9}$ Plasma aldosterone was measured by the coat-a-count radioimmunoassay procedure (Diagnostic Products Corporation, Los Angeles, California, USA). Plasma vasopressin (AVP) was measured on extracted samples by radioimmunoassay according to the methods described by Woods and Johnston. ${ }^{10}$ Plasma atrial natriuretic peptide (ANP) levels were determined by a radioimmunoassay on unextracted plasma (Casley, personal communications).

\section{Statistical Analysis}

One way analysis of variance was used to examine differences in demographic data in the 3 study groups and mean change in bladder pressure between the 3 study groups.

Two way analysis of variance extracting within personal changes and between group differences was performed to examine blood pressure alterations. Pairwise comparisons were made for analysis of simple effects provided the $F$ value in the analysis of variance achieved statistical significance.

A similar analysis was performed to examine changes in pressor hormone levels before and during bladder distension between study groups.
Linear correlation was performed to examine relationships between changes in bladder pressure, heart rate, blood pressure and pressor hormones in each study group.

All values presented are the mean \pm standard deviation.

\section{Results}

There were no significant differences between the 3 groups studied in age (high spinal cord injury $34.6 \pm 16.3$ years, low spinal cord injury $38.8 \pm 14.9$, control subjects $43.3 \pm 5.0, p=0.12)$; time since spinal cord injury (high spinal cord injury 5.2 \pm 3.1 years, low spinal cord injury $11.6 \pm 8.4$, $p=0.06$ ); or sex (high spinal cord injury 8 males, one female; low spinal cord injury 7 males, no females; control subjects 6 males, one female). Increases in bladder pressure induced at the time of bladder filling were not significantly different in the 3 study groups (Table 1).

Finapres blood pressure recordings at baseline were somewhat lower than manual readings (systolic blood pressure: Finapres $137 \pm 19 \mathrm{~mm} \mathrm{Hg}$, manual $139 \pm 19 \mathrm{~mm} \mathrm{Hg}$, $r=0.89, p>0.1$; diastolic blood pressure: Finapres $69 \pm 12 \mathrm{~mm} \mathrm{Hg}$, manual $75 \pm$ $12 \mathrm{~mm} \mathrm{Hg}, r=0.69, p>0.05)$.

Systolic blood pressure, diastolic blood pressure and heart rate measurements by the Finapres technique before and during bladder distension in the 3 study groups are summarised in Figure 1.

There were no significant differences in systolic blood pressure at baseline between the 3 groups but there were significant differences in baseline diastolic blood pressure, the 3 groups with the high spinal lesion group having the lowest diastolic blood pressure $(p=0.044)$. There were also significant differences in the 3 groups at baseline for heart rate $(p<0.001)$, with the high spinal lesion group having the lowest heart rate and the low spinal lesion group having the highest heart rate. Rises in systolic and diastolic blood pressure during bladder distension were significant in the high spinal lesion group $(p<0.001, p<0.001)$, but not in the low spinal lesion group or the normal controls. In the high spinal lesion group, the 
Table 1 Bladder pressure and vasoactive hormone responses to bladder distension in the 3 study groups (mean \pm standard deviation). There were no significant differences in bladder pressure rise in response to bladder distension between the 3 study groups. There were no significant differences in baseline levels of any hormone between the 3 groups. There were no significant differences in plasma levels of noradrenaline, renin concentration, vasopressin or atrial natriuretic peptide during bladder distension in comparison to baseline in any of the study groups. Plasma aldosterone levels fell significantly following bladder distension in the low spinal lesion and control groups (low spinal: $p=0.026$; control: $p=0.013$ ), but not in the high spinal lesion group

\begin{tabular}{|c|c|c|c|c|c|c|}
\hline & $\begin{array}{c}\text { Bladder } \\
\text { pressure } \\
\text { rise } \\
\left(\mathrm{cm} \mathrm{H} \mathrm{H}_{2} \mathrm{O}\right)\end{array}$ & $\begin{array}{c}\text { Plasma } \\
\text { noradrenaline } \\
(\mathrm{mmol} / \mathrm{L})\end{array}$ & $\begin{array}{c}\text { Plasma } \\
\text { renin } \\
\text { activity } \\
(\mathrm{ng} / \mathrm{ml} / \mathrm{hr})\end{array}$ & $\begin{array}{l}\text { Plasma } \\
\text { aldosterone } \\
(\mathrm{ng} / \mathrm{dl})\end{array}$ & $\begin{array}{c}\text { Plasma } \\
\text { vasopressin } \\
(\mathrm{pg} / \mathrm{dl})\end{array}$ & $\begin{array}{c}\text { Plasma } \\
\text { atrial } \\
\text { natriuretic } \\
\text { peptide } \\
(\mathrm{pg} / \mathrm{ml})\end{array}$ \\
\hline \multicolumn{7}{|l|}{$\begin{array}{l}\text { Control } \\
\text { subjects }\end{array}$} \\
\hline & - & $2.74 \pm 3.02$ & $1.21 \pm 0.59$ & $12.7 \pm 2.5$ & $4.82 \pm 1.48$ & $202 \pm 79$ \\
\hline Distension & $57 \pm 27$ & $2.58 \pm 2.82$ & $0.98 \pm 0.35$ & $9.1 \pm 2.7^{*}$ & $5.48 \pm 2.40$ & $187 \pm 81$ \\
\hline \multicolumn{7}{|l|}{$\begin{array}{l}\text { High spinal } \\
\text { cord injury } \\
\text { patients }\end{array}$} \\
\hline $\begin{array}{l}\text { Baseline } \\
\text { Bladder }\end{array}$ & - & $0.72 \pm 0.34$ & $2.25 \pm 0.79$ & $21.7 \pm 11.5$ & $5.70 \pm 1.87$ & $142 \pm 35$ \\
\hline Distension & $60 \pm 25$ & $1.25 \pm 1.09$ & $1.98 \pm 0.61$ & $17.1 \pm 7.2^{* *}$ & $5.11 \pm 1.54$ & $165 \pm 104$ \\
\hline \multicolumn{7}{|l|}{$\begin{array}{l}\text { Low spinal } \\
\text { cord injury } \\
\text { patients }\end{array}$} \\
\hline $\begin{array}{l}\text { Baseline } \\
\text { Bladder }\end{array}$ & - & $3.38 \pm 2.19$ & $2.71 \pm 1.75$ & $16.6 \pm 7.6$ & $6.28 \pm 4.23$ & $143 \pm 60$ \\
\hline Distension & $48 \pm 20$ & $3.02 \pm 1.23$ & $2.34 \pm 2.13$ & $11.6 \pm 4.0$ & $4.66 \pm 1.27$ & $115 \pm 63$ \\
\hline
\end{tabular}

rise in blood pressure was associated with a significant fall in heart rate following bladder distension $(p=0.029)$. There was no significant change in heart rate in the other 2 groups.

Mean ( \pm standard deviation) of plasma noradrenaline levels, aldosterone levels, plasma renin concentration, vasopressin levels and atrial natriuretic peptide levels before and during bladder distension in the 3 study groups are presented in Table 1 . Overall there were no significant differences in baseline plasma noradrenaline levels between the 3 groups, although baseline plasma noradrenaline levels appeared lowest in the high spinal lesion group. There was a small, non significant rise in plasma noradrenaline in the high spinal lesion group following bladder distension, while plasma noradrenaline levels tended to fall following bladder distension in the other 2 study groups. No significant correlations were found between changes in plasma noradrenaline following bladder distension in the high spinal lesion group and changes in systolic or diastolic blood pressure or change in bladder pressure. However, a significant correlation was noted at baseline between serum noradrenaline levels and heart rate in all study subjects $(r=0.891$, $p<0.01$ ), but not when the 3 groups were analysed individually or during bladder distension.

There were no significant differences in baseline levels of plasma renin concentration between the 3 study groups. In addition, plasma renin concentration did not significantly change following bladder 

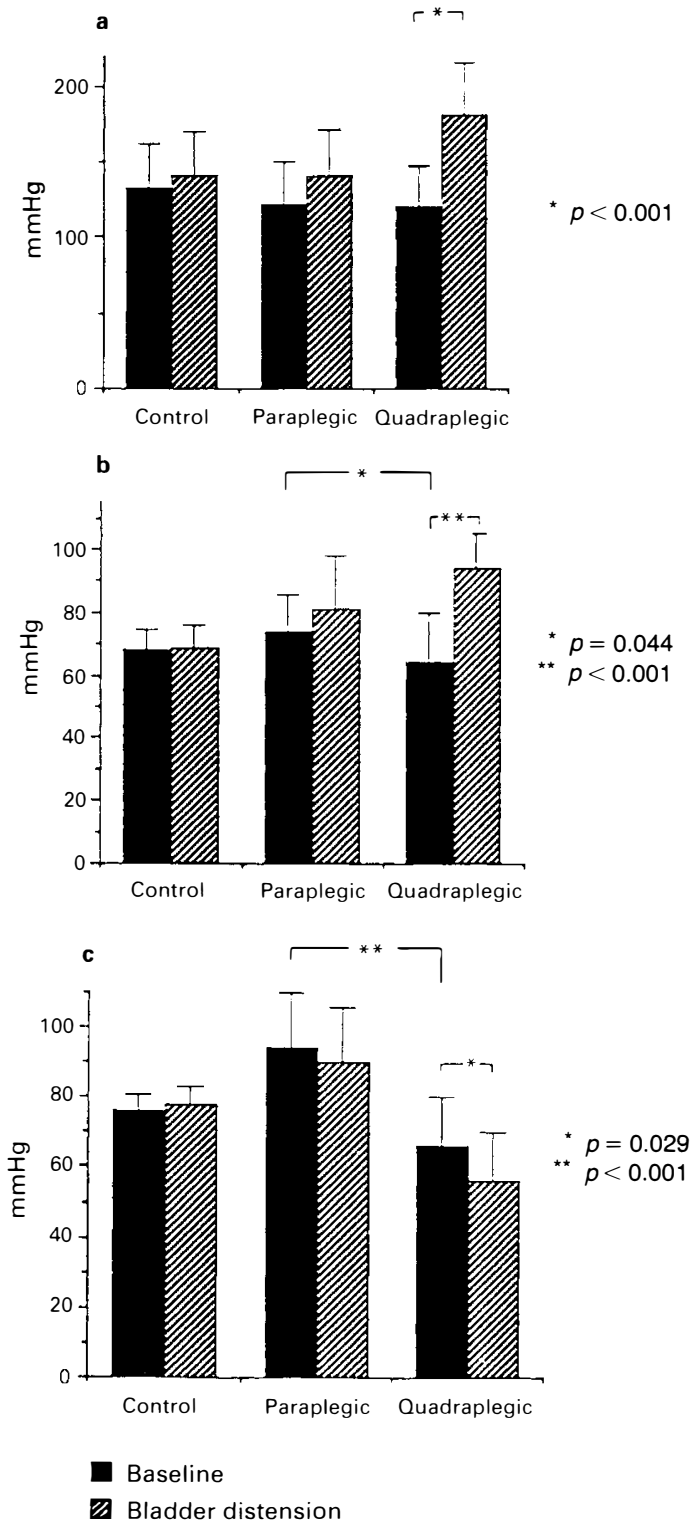

Figure 1 Systolic blood pressure (a), diastolic blood pressure (b) and heart rate measurements (c) (mean \pm standard deviation) before and during bladder distension in the 3 study groups. There were significant differences in diastolic blood pressure $(p=0.044)$ and heart rate $(p<0.001)$ but not systolic blood pressure at baseline between the 3 study groups. There was a significant rise in blood pressure and fall in heart rate during bladder distension in the high spinal lesion group (systolic blood pressure: $p<0.001$ : diastolic blood pressure: $p<0.001$; heart rate: $p=0.029$ ) but not in the low spinal lesion group or the normal controls. distension in any of the study groups, although all groups showed a trend for plasma renin concentration to fall. While there were no significant differences in baseline levels of plasma aldosterone, plasma vasopressin or atrial natriuretic peptide between the 3 study groups, plasma aldosterone levels fell significantly following bladder distension in the low spinal lesion and control groups (low spinal: $p=0.026$; control: $p=0.013$ ). Plasma aldosterone levels also tended to fall following bladder distension in the high spinal lesion group, but this fall did not reach statistical significance. While plasma vasopressin levels tended to fall during bladder distension in the high and low spinal lesion groups, these falls did not reach statistical significance. Plasma vasopressin levels tended to rise during bladder distension in control subjects, but again this was not statistically significant. Plasma atrial natriuretic peptide levels did not change significantly in any of the study groups.

\section{Discussion}

The present study documents blood pressure responses to bladder distension in normal subjects and spinal patients.

As has been previously demonstrated by Wurster and Randall, ${ }^{11}$ there was a significant difference in the systemic blood pressure response to bladder distension in high and low spinal lesion patients. The extent of the systolic and diastolic blood pressure rise in high spinal patients in the present study was of the same order as that reported in their study and in the study of Mathias, et al. ${ }^{12}$

The rise in blood pressure in the normal control group was somewhat less marked than that obtained by Fagius and Karhuvaara $^{13}$ where bladder overdistension and abdominal pain probably contributed to an exaggerated pressor response. It is important to note that none of the normal control subjects or low spinal lesion patients undergoing urodynamics in the present study experienced abdominal pain from bladder distension and therefore a pain induced rise in blood pressure is unlikely to have occured. 
Mathias et $\mathrm{al}^{12,13}$ have previously demonstrated increases in plasma noradrenaline corresponding to increased blood pressure during suprapubic bladder tapping in high spinal cord lesion patients. Fagius and Karhuvaara $^{14}$ demonstrated increased sympathetic activity following bladder distension in normal control subjects while Stjernberg, et $a^{15}$ noted increased sympathetic activity in spinal patients following suprapubic bladder tapping when sympathetic function was assessed by direct microneuroelectrode recordings of muscle-nerve sympathetic activity. This technique may be a more sensitive index of short term increases in sympathetic activity than plasma noradrenaline sampling which is dependent on neuronal activation, noradrenaline release from nerve terminals and subsequent spillover into the systemic circulation.

In the present study, there was a slight increase in plasma noradrenaline in the high spinal lesion group (in comparison to to a tendency for a fall in plasma noradrenaline in the other 2 study groups); however this rise did not reach statistical significance. It is possible that more frequent sampling of plasma noradrenaline levels may may have demonstrated a statistically significant rise. Alternatively, slow bladder filling may not produce as dramatic a sympathetic stimulas as suprapubic bladder tapping. The high spinal lesion patients involved in the present study had not experienced recent episodes of autonomic hyperreflexia. It is unclear whether patients studied by Mathias et $a l^{12,13}$ had experienced recent episodes of autonomic hyperreflexia, but a difference in study groups of this type may explain the differences in the results of the 2 studies as may a type II error.

The lack of change in plasma renin and aldosterone levels suggests that the renin angiotensin system does not play a major role in bladder induced elevations in blood pressure in spinal cord injury patients. Indeed, plasma renin and aldosterone tended to fall with bladder distension. This is in keeping with the findings of Nanninga et $a l^{16}$ and Mathias $e a{ }^{17}$ who noted a fall in plasma renin following bladder distension in patients with autonomic hyperreflexia. The reduction in plasma renin levels with blad- der distension may be a reflex fall in response to elevated blood pressure.

The lack of alteration of vasoactive hormone levels in the high spinal lesion group in response to bladder distension despite large increases in blood pressure, suggests that these rises are neurally mediated, supporting the postulate put forward by Mathias et $a l^{12}$ among others. Ascending sympathetic activity in the decentralised cord may not be dampened by descending inhibitory supraspinal pathways that would normally suppress this activity. This in turn may result in increased reflex sympathetic stimulation of adrenoceptors on the splanchnic vasculature below the level of injury leading to systemic hypertension.

In summary, the present study has demonstrated major differences in blood pressure response to bladder distension in high spinal lesion patients (without current problems of autonomic hyperreflexia) in comparison to patients with low spinal transection and normal subjects. These results support earlier findings ${ }^{17,18}$ of the relationship between increases in urinary bladder pressure and elevations in blood pressure in man which is normally supressed when spinal pathways are intact. There were no changes in hormones measured during the period of bladder distension in comparison to baseline measurements that could implicate humoral mechanisms in the blood pressure rises experienced by the high spinal lesion patients.

These findings therefore support earlier suggestions that neural pathways below the level of cord transection with sympathetic efferents, in association with increased vascular sensitivity, ${ }^{12,13}$ most likely mediate blood pressure elevations in response to bladder distension in these patients.

\section{Acknowledgements}

Dr Krum is a postgraduate medical research scholar of the National Health and Medical Research Council of Australia. This study was supported by a grant from the Austin Hospital Medical Research Foundation and National Health and Medical Research Council Programme Grant. 


\section{References}

1 Clarke SJ, Syme RA (1978) Urodynamic evaluation in the management of the spinal injury patient. Aust NZ J Surg 48: 184-188.

2 Penaz J (1973) Photoelectric measurement of blood pressure, volume and flow in the finger. In: Albert R, Vet W, Helbeg W, eds. Digest of the 10th Conference on Medical and Biological Engineering: 104.

3 Wesseling KM, De Wit B, Settels JJ, Klawer WH (1982) On the indirect registration of finger blood pressure after Penaz. Funktional Biological Medicine 1: 245-250.

4 Smith NT, Wesseling KW, De Wit B (1986) Evaluation of two prototype devices producing noninvasive pulsatile calibrated blood pressure from a finger. J Clin Monitoring 1: 17-29.

5 Parati G, Casadei R, Gropelli A, DI Rienzo M, Mancia G (1989) Comparison of finger and intra-arterial blood pressure monitoring at rest and during laboratory testing. Hypertension 13: 647-655.

6 Howes LG, Summers RJ, Rowe PR, Louis W (1983) The simultaneous determination of 3,4-dihydroxy-phenylethylene glycol, 3,4-dihydroxyphenylacetic acid and catecholamines in brain tissue by high performance liquid chromatography with electrochemical detection. Neurosci Letters 38: 327.

7 Howes LG, Rowe PR (1987) Combined cation exchange-alumina extraction of [3H] noradrenaline for the determination of $[3 \mathrm{H}]$ noradrenaline plasma kinetics in man. Scand J Clin Lab Investig 47: 749-750.

8 Jackman GP, Rowe P, Howes LG (1990) Simplified procedure to remove radiolabelled contaminants after $[3 \mathrm{H}]$ norepinephrine infusions. Clin Chem 36: 1-3.

9 Johnston CI, Mendelsohn FAO, Casley DJ (1969) Plasma renin determinations employing a radioimmunoassay for angiotensin I. Proc Aust Soc Med Res 2: 271.

10 Woods RL, Johnston CI (1983) Contribution of vasopressin to the maintenance of blood pressure during dehydration. Am J Physiol 245: F615-F621.

11 Wurster RD, Randall WC (1975) Cardiovascular responses to bladder distension in patients with spinal transection. Am J Physiol 228: 1288-1292.

12 Mathias CJ, Frankel HL, Christensen NJ, Spalding JMK (1986) Enhanced pressor responses to noradrenaline in patients with cervical spinal cord transection. Brain 99: 757-770.

13 Mathias CJ, Christensen NJ, Corbett JL, Frankel HL, Spalding JMK (1976) Plasma catecholamines during paroxysmal neurogenic hypertension in quadriplegic man. Circulation Res 39: 204-208.

14 Fagius J, Karhuvaara S (1989) Sympathetic activity and blood pressure increases with bladder distension in humans Hypertension 14: 511-517.

15 Stjernberg, Blumberg and Wallin (1986) Sympathetic activity in man after spinal cord injury. Outflow to muscle below lesion. Brain 109: 695-715.

16 Nanninga JB, Rosen JS, Krumlovsky F (1976) Effect of autonomic hyperreflexia on plasma renin. Urol 7: $638-640$.

17 Mathias CJ, Frankel HL, Davies IB, James VHT, Peart WS (1981) Renin and aldosterone release during sympathetic stimulation in tetraplegia. Clin Sci 60: 399-404.

18 Corbett JL, Frankel HL, Harris PJ (1971) Cardiovascular reflex responses to cutaneous and visceral stimuli in spinal man. $J$ Physiol 215: 395-409. 